\title{
Electromagnetic neutrino interactions in elastic neutrino-proton scattering
}

\author{
Konstantin Kouzakov, ${ }^{a}$ Fedor Lazarev ${ }^{b, *}$ and Alexander Studenikin ${ }^{b, c}$ \\ ${ }^{a}$ Department of Nuclear Physics and Quantum Theory of Collisions, Faculty of Physics, Lomonosov \\ Moscow State University, \\ Moscow 119991, Russia \\ ${ }^{b}$ Department of Theoretical Physics, Faculty of Physics, Lomonosov Moscow State University, \\ Moscow 119991, Russia \\ ${ }^{c}$ Joint Institute for Nuclear Research, \\ Dubna 141980, Moscow Region, Russia \\ E-mail: kouzakov@srd.sinp.msu.ru, fedek302@gmail.com, \\ studenik@srd.sinp.msu.ru
}

\begin{abstract}
We develop a basic theoretical apparatus for the search of electromagnetic neutrino interactions in experiments on elastic neutrino-nucleus scattering. Using our approach developed for the case of elastic neutrino-electron collisions, we take into account all electromagnetic form factors of massive neutrinos. In this contribution we focus on elastic neutrino-proton scattering, which can be important for studying supernova neutrinos in experiments, for example, such as JUNO. In our consideration we take into account all electromagnetic form factors not only of a neutrino, but of a proton as well.
\end{abstract}

40th International Conference on High Energy physics - ICHEP2020

July 28 - August 6, 2020

Prague, Czech Republic (virtual meeting)

\footnotetext{
${ }^{*}$ Speaker
} 
One interesting consequence of nonzero neutrino masses is that neutrinos can have nonzero electromagnetic properties [1]: millicharges and charge radii, electric, magnetic and anapole moments. These properties can manifest themselves in elastic neutrino-electron scattering [2] and, in particular, in coherent elastic neutrino-nucleus scattering (CEvNS) [3] that recently has become accessible for experimental investigation [4]. The simplest nuclear target for $\mathrm{CE} v \mathrm{NS}$ is a proton. In this contribution we consider the process where an ultrarelativistic neutrino with energy $E_{v}$ originates from a source (reactor, accelerator, the Sun, etc.) and elastically scatters on a proton in a detector at energy-momentum transfer $q=(T, \mathbf{q})$. If the neutrino is born in the source in the flavor state $\left|v_{\ell}\right\rangle$, then its state in the detector is $\left|v_{\ell}(L)\right\rangle=\sum_{k=1}^{3} U_{\ell k}^{*} \exp \left(-i \frac{m_{k}^{2}}{2 E_{v}} \mathcal{L}\right)\left|v_{k}\right\rangle$, where $\mathcal{L}$ is the source-detector distance. We assume the target proton to be free and at rest in the lab frame. The matrix element of the transition $v_{\ell}(L)+p \rightarrow v_{j}+p$ due to weak interaction is given by

$$
\mathcal{M}_{j}^{(w)}=\frac{G_{F}}{\sqrt{2}} U_{\ell j}^{*} e^{-i \frac{m_{j}^{2}}{2 E_{\nu}}} \mathcal{L}_{\bar{u}_{j, \lambda^{\prime}}^{(\nu)}}\left(k^{\prime}\right) \gamma^{\lambda}\left(1-\gamma^{5}\right) u_{j, \lambda}^{(v)}(k) \bar{u}_{s^{\prime}}^{(p)}\left(p^{\prime}\right)\left(g_{V} \gamma^{\lambda}-g_{A} \gamma^{\lambda} \gamma^{5}\right) u_{s}^{(p)}(p) .(1)
$$

where $g_{V}^{p}=1 / 2-2 \sin ^{2} \theta_{W}, g_{A}^{p}=1 / 2, g_{V}^{n}=-1 / 2, g_{A}^{n}=-1 / 2 . \bar{u}_{j, \lambda^{\prime}}^{(\nu)}\left(k^{\prime}\right)=u_{j, \lambda^{\prime}}^{(\nu) \dagger}\left(k^{\prime}\right) \gamma^{0}$, where $u_{j, \lambda}^{(\nu)}(k)$ is the bispinor amplitude of the massive neutrino state $\left|v_{j}\right\rangle$ with four-momentum $k$ and spin $\lambda$. $u_{s}^{(p)}(p)$ is the bispinor amplitude of the proton with four-momentum $p$ and spin $s$. The matrix element due to electromagnetic interaction is given by

$$
\mathcal{M}_{j}^{(\gamma)}=-\frac{4 \pi \alpha}{q^{2}} \sum_{k=1}^{3} U_{\ell k}^{*} e^{-i \frac{m_{k}^{2}}{2 E_{\nu}}} \mathcal{L}_{\bar{u}_{j, \lambda^{\prime}}^{(v)}}\left(k^{\prime}\right) \Lambda_{\mu}^{j k}(q) u_{k, \lambda}^{(v)}(k) \bar{u}_{s^{\prime}}^{(N)}\left(p^{\prime}\right) \Gamma^{\mu}(-q) u_{s}^{(N)}(p, s),
$$

where $\Lambda_{\mu}^{j k}(q)$ and $\Gamma^{\mu}(q)$ are electromagnetic vertexes for the neutrino and proton, respectively,

$$
\begin{aligned}
\Lambda_{\mu}^{f i}(q) & =\left(\gamma_{\mu}-q_{\mu} q / q^{2}\right)\left[f_{Q}^{f i}\left(q^{2}\right)+f_{A}^{f i}\left(q^{2}\right) q^{2} \gamma_{5}\right]-i \sigma^{\mu v} q_{v}\left[f_{M}^{f i}\left(q^{2}\right)+i f_{E}^{f i}\left(q^{2}\right) \gamma_{5}\right] \\
\Gamma^{\mu}(q) & =\gamma^{\mu} F_{Q}\left(q^{2}\right)+\left(\gamma^{\mu}-q^{\mu} q / q^{2}\right) \gamma_{5} F_{A}\left(q^{2}\right) q^{2}-i \sigma^{\mu v} q_{v} F_{M}\left(q^{2}\right)+\sigma^{\mu v} q_{v} \gamma_{5} F_{E}\left(q^{2}\right) .
\end{aligned}
$$

When evaluating the cross section, we neglect the neutrino masses. Since the final massive state of the neutrino is not resolved in the detector, the differential cross section measured in the scattering experiment is given by

$$
\frac{d \sigma}{d T}=\frac{|\mathcal{M}|^{2}}{32 \pi E_{v}^{2} m_{N}},
$$

with the following absolute matrix element squared:

$$
|\mathcal{M}|^{2}=\sum_{j=1}^{3}\left|\mathcal{M}_{j}^{(w)}+\mathcal{M}_{j}^{(\gamma)}\right|^{2}
$$

where, as usual, averaging over initial and summing over final spin polarizations is assumed. The differential cross section can be presented as a sum of the helicity-preserving (hp) and helicityflipping (hf) parts: $\frac{d \sigma}{d T}=\frac{d \sigma_{\mathrm{hp}}}{d T}+\frac{d \sigma_{\mathrm{hf}}}{d T}$. Below we present these parts in the case of a zero proton anapole form factor and in the limit of a small energy transfer $T$ :

$$
\begin{aligned}
\frac{d \sigma_{\mathrm{hp}}}{d \Omega}= & \frac{G_{F}^{2} m_{p}}{2 \pi}\left[\left(C_{Q}+2 \operatorname{Re} C_{Q \& A}+g_{A}^{2}\right)+\left(C_{Q}-2 \operatorname{Re} C_{Q \& A}+g_{A}^{2}\right)\left(1-\frac{2 T}{E}\right)\right. \\
& \left.+\left(g_{A}^{2}-C_{Q}\right) \frac{m_{p} T}{E^{2}}+\frac{T}{2 m_{p}}\left(C_{M}-C_{E}\right)+4 \frac{T}{E} \operatorname{Re} C_{A \& M}\right],
\end{aligned}
$$




$$
\frac{d \sigma_{\mathrm{hf}}}{d T}=\frac{\pi \alpha^{2}}{m_{e}^{2}}\left|\mu_{\nu}\right|^{2}\left[\left(\frac{1}{T}-\frac{1}{E}\right)\left|F_{Q}\right|^{2}-\frac{T}{2 E^{2}} \operatorname{Re}\left(F_{q} F_{M}^{*}\right)+\frac{\left(1-\frac{T}{E}\right)-\frac{m_{N} T}{E^{2}}}{4 m_{N}}\left|F_{M}\right|^{2}-\frac{\left(1-\frac{T}{E}\right)}{4 m_{N}}\left|F_{E}\right|^{2}\right]_{(7)},
$$

where

$$
\begin{aligned}
C_{Q} & =\sum_{j}\left|\sum_{k} U_{\ell k}^{*} \exp \left(-i \frac{m_{k}^{2}}{2 E} \mathcal{L}\right)\left(\delta_{j k} g_{V}-F_{Q} Q_{j k}\right)\right|^{2}, \\
Q_{j k} & =\frac{2 \sqrt{2} \pi \alpha}{G_{F} q^{2}}\left(e_{j k}+\frac{q^{2}}{6}\left\langle r^{2}\right\rangle_{j k}\right), \quad\left\langle r^{2}\right\rangle_{j k}=r_{j k}^{2}-6 a_{j k}, \\
C_{Q \& A} & =-g_{A} \sum_{j, k} U_{\ell j} U_{\ell k}^{*} \exp \left(i \frac{\delta m_{j k}^{2}}{2 E} \mathcal{L}\right)\left(\delta_{j k} g_{V}-F_{Q} Q_{j k}\right), \quad \delta m_{j k}^{2}=m_{j}^{2}-m_{k}^{2}, \\
C_{M} & =\sum_{j}\left|\sum_{k} U_{\ell k}^{*} \exp \left(-i \frac{m_{k}^{2}}{2 E} \mathcal{L}\right) F_{M} Q_{j k}\right|^{2}, \\
C_{E} & =\sum_{j}\left|\sum_{k} U_{\ell k}^{*} \exp \left(-i \frac{m_{k}^{2}}{2 E} \mathcal{L}\right) F_{E} Q_{j k}\right|^{2}, \\
C_{A \& M} & =-g_{A} \sum_{j, k} U_{\ell k} U_{\ell j}^{*} \exp \left(-i \frac{\delta m_{j k}^{2}}{2 E} \mathcal{L}\right) F_{M}^{*} Q_{j k}, \\
\left|\mu_{v}\right|^{2} & =\sum_{j}\left|\sum_{k} U_{\ell k}^{*} \exp \left(i \frac{m_{k}^{2}}{2 E} \mathcal{L}\right)\left(\mu_{j k}-i \epsilon_{j k}\right)\right|^{2} .
\end{aligned}
$$

It should be noted that the derived expressions for the elastic neutrino-proton scattering account for the electric dipole moment of a proton. These formulas can be readily applied to elastic neutrino-neutron scattering, upon substituting the proton form factors with the neutron ones.The expressions obtained can be used to analyze and interpret the data of neutrino scattering in detectors containing hydrogen [5], as well as to study the propagation and interaction in dense nuclear matter, for example, in neutron stars, supernovas, etc.

This work was supported by the Russian Foundation for Basic Research under Grant No. 20-52-53022-GFEN-a.

\section{References}

[1] C. Giunti and A. Studenikin, Rev. Mod. Phys. 87 (2015) 531 [arXiv:1403.6344].

[2] K. A. Kouzakov and A. I. Studenikin, Phys. Rev. D 96 (2017) 099904 [arXiv:1703.00401].

[3] M. Cadeddu, C. Giunti, K. A. Kouzakov, Y. F. Li, A. I. Studenikin, and Y. Y. Zhang, Phys. Rev. D 98 (2018) 113010 [arXiv:1810.05606].

[4] D. Akimov et al. (COHERENT), Science 357 (2017) 1123 [arXiv:1708.01294].

[5] F. An et al., J. Phys. G 43 (2016) 030401 [arXiv:1507.05613]. 